\title{
VECTOR DIFFRACTION THEORY AND COHERENT TRANSITION RADIATION INTERFEROMETRY IN ELECTRON LINACS*
}

\author{
T. J. Maxwell, C. L. Bohn, D. Mihalcea, Northern Illinois University, DeKalb, IL 60115, U.S.A. \\ P. Piot, Northern Illinois University, DeKalb, IL 60115, and Fermilab, Batavia, IL 60510, U.S.A.
}

\section{Abstract}

Electrons impinging on a thin metallic foil produce small bursts of transition radiation (TR) as they cross the boundary from one medium to the next. A popular diagnostic application is found for compact electron bunches. In this case they will emit radiation more or less coherently with an enhancement of the intensity on wavelengths comparable to or larger than the bunch size, generating coherent transition radiation (CTR). Several detailed analytical descriptions have been proposed for describing the resulting spectral distribution, often making different simplifying assumptions. Given that bunches tenths of millimeters long can generate measurable spectra into the millimeter range, concern may arise as to weak diffraction effects produced by optical interference devices containing elements with dimensions in the centimeter range.

\section{INTRODUCTION}

When a relativistic electron impinges on a foil it will emit transition radiation (TR). There are many ways to model this effect, the classic model being that of the Ginzburg-Frank formula [1] by solving Maxwell's equations at a metallic boundary of infinite extent.

From here, if one calculates the resulting intensity for a bunch they find there is a coherent $\mathrm{N}$-squared enhancement related to the Fourier transform of the longitudinal bunch distribution dominating the incoherent by a factor of $N$, where $N$ is the number of particles per bunch. By collecting this coherent transition radiation (CTR) and interfering it with a path-delayed image of itself, one can generate the auto-correlation of the intensity and infer the longitudinal bunch distribution. This is typically performed with a Michelson interferometer such as that at the Fermilab/NICADD photoinjector laboratory. The elements of this standard interferometer include an acceptance collimating parabolic mirror with $152.4 \mathrm{~mm}$ focal length with $76.2 \mathrm{~mm}$ $\mathrm{OD}$, as well as subsequent beam splitters and mirrors of $76.2 \mathrm{~mm}$ OD.

The aim of our calculation is to take the effects of finite laboratory equipment into account while at the same time having a viable way to model the transition radiation. The assumption is that if there are any noticeable diffraction effects as the CTR propagates through the interferometer, some radiation may leak past the various optical elements and escape detection. If significant in the millimeter to sub-millimeter range, this would effectively alter the

\footnotetext{
*Work supported by US. Department of Energy, under Contract No.
} DE-FG02-06ER41435 with Northern Illinois University frequency response of the interferometer being used.

To meet these demands, we examine the use of the virtual quanta method that represents the relativistic electrons by their Fourier components as in [2]. We can then employ a vector diffraction technique satisfying Maxwell's equations [4] to analyze the effects of the finite elements of the system assuming them to be ideal optical elements over arbitrary regions of 3D space with no paraxial approximations.

\section{VECTOR DIFFRACTION THEORY}

Equation (1) is the formula used for computing the electric and magnetic fields at an arbitrary point in space, as adapted from reference [4] to include terms second order in $R$. Assuming an otherwise field-free region with a source field defined at a given frequency in a finite area somewhere in space, the fields in the subsequent region are given by:

$$
\begin{aligned}
& \vec{E}(\vec{r})=\frac{-i}{2 \lambda^{2}} \iint_{\text {Aperture }}\{[1+\hat{R} \cdot \hat{n}] \vec{E}(\vec{R})-[\hat{R} \cdot \vec{E}(\vec{R})][\hat{R}+\hat{n}]\}[\exp (i k R) / R] d A \\
& +\frac{1}{2 \lambda^{2}} \iint_{\text {Aperture }}[(\hat{R} \cdot \hat{n}) \vec{E}(\vec{R})-(\hat{R} \cdot \vec{E}(\vec{R})) \hat{n}]\left[\exp (i k R) / k R^{2}\right] d A
\end{aligned}
$$

Where $\vec{r}$ is the point of observation, $\vec{R}$ is the relative vector from the source point to the point of observation, and $\vec{n}$ is the unit Poynting vector at the source. The magnetic field expression is identical with $\vec{B}$ exchanged everywhere for $\vec{E}$. These parameters are all tracked easily when evaluated computationally using a mesh method. Tracking geometrical meshes with an associated EM field evaluated everywhere over that surface mesh allows one to map from one surface to the next, calculate $\hat{n}$, and then integrate to the next surface. The use of a vector formulation such as this is critical as methods only accounting for the electric field component will not allow for a second diffraction pattern to be calculated, such as for the reflection from a finite mirror. In the case of a reflection, the image at a source is "projected" to the mirror surface using the above integral, the reflection conditions at a metallic mirror are performed on the orthogonal set of the EM field at the point, then projected to a viewing area of interest.

\section{SINGLE SURFACE BENCHMARKING}

Calculations for simple, well-known diffraction patterns were performed to verify the validity of the full 3D implementation of (1). In all cases for both near-field and far-field effects of circular and rectangular apertures, the 
resulting patterns along cuts matched with theoretically predicted patterns. Figure 1 shows the example of a circular aperture shown in both the near- and far-fields. The top and bottom images are taken along the plane transverse to the aperture with parallel plane cuts shown center.

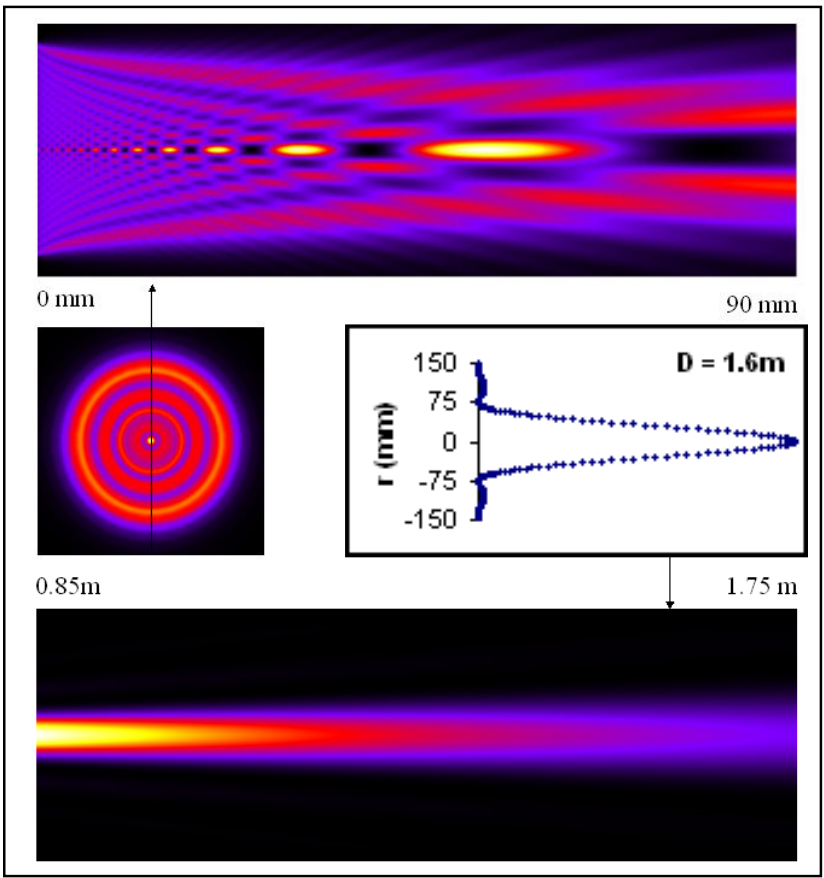

Figure 1: Intensities (arb. units) along the transverse plane in the near field (top) and far field (bottom), with example cuts at $15 \mathrm{~mm}$ and $1.6 \mathrm{~mm}$ (center) for circular aperture O.D. $=25.4 \mathrm{~mm}$ at $\lambda=1 \mathrm{~mm}$

It is worthwhile noting that for large viewing areas parallel to the aperture that the total integrated intensity over the aperture was very well conserved at the viewing surface. This was, however, affected slightly by the size of the viewing region and the resolution of the mesh being used. The latter reflects the loss of EM surface detail at low resolutions, but integrated values were found to converge quickly enough to not make very highresolution calculations necessary. An adjustable mesh method may be employed in the future to automatically enhance detail in regions that vary greatly. With the intent of the final simulation being to track accepted total intensities from one surface to the next, this conservation and convergence analysis is critical at every stage.

\section{PARABOLIC MIRROR}

The method prescribed above was used to image a point "photon" up into the mirror from the focal point and reflected into the forward direction of the mirror (Figure 2). Comparison of the resulting pattern to a known standard is difficult and pending for this geometry, though it was at least found to be self-consistent. We note qualitatively, that the line of central max for the point source appear as though the point source was deflected away along the curvature of the mirror. Aside from the natural bilateral symmetry of the field, we also note the circular aperture-like modulation of the projection due to the circular section cut of the mirror.

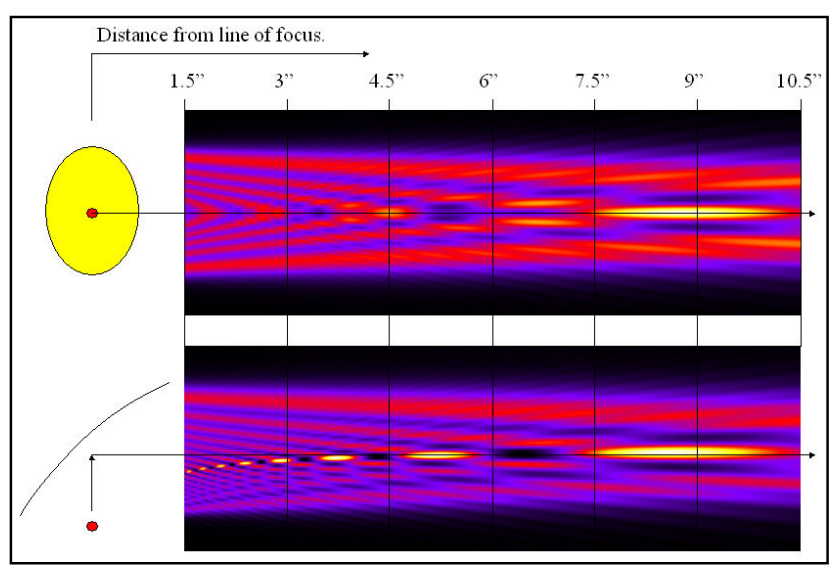

Figure 2: Intensity distribution (arb. units) along planar cuts transverse to the axis from the 90-degree deflection point of the mirror for $\lambda=1 \mathrm{~mm}$, E.F.L. $=152.4 \mathrm{~mm}$, O.D. $=50.8 \mathrm{~mm}$ with a point source "photon" at focus.

Analysis of the same mirror in focusing mode is shown in Figure 3. In this case, a Gaussian laser beam field was projected onto the surface of the mirror then reflected into the focal point of the mirror. We note that maximum peak intensity is located at the focal point of the mirror, though a bilaterally distorted transverse distribution was observed (not shown here) as was observed in [2].

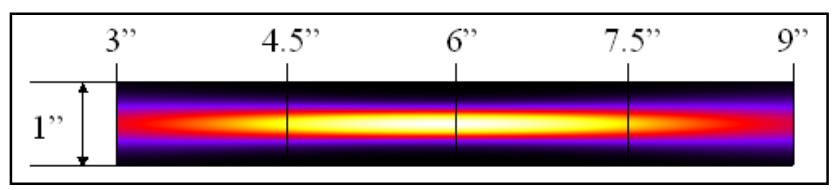

Figure 3: Intensity distribution (arb. units) along planar cut transverse to the axis from the 90-degree deflection point of the mirror for $\lambda=1 \mathrm{~mm}$, E.F.L. $=152.4 \mathrm{~mm}$, O.D. $=50.8 \mathrm{~mm}$ with a Gaussian laser source, RMS radius $=$ $6.35 \mathrm{~mm}$ reflected into focus. 


\section{TRANSITION RADIATION BY VIRTUAL QUANTA}

An analysis of transition radiation of one electron as well as the coherent radiation of multiple electrons has just begun. The approach outlined above is applied using the initial EM field source as the frequency components of a relativistic electron. By working with single frequencies, we have the freedom to use the optical approach outlined above to propagate the field through the instrument. For a relativistic electron traveling in the $z$-direction, the time Fourier transformed field components are found to be:

$$
\begin{aligned}
& \widetilde{E}_{r}(r, z, \omega)=\frac{q \omega}{(2 \pi)^{3 / 2} \varepsilon_{0} \beta^{2} c^{2} \gamma} K_{1}\left(\frac{\omega}{\beta c \gamma} r\right) \exp \left(\frac{i \omega}{\beta c} z\right) \\
& \widetilde{E}_{z}(r, z, \omega)=\frac{-i q \omega}{(2 \pi)^{3 / 2} \varepsilon_{0} \beta^{2} c^{2} \gamma^{2}} K_{0}\left(\frac{\omega}{\beta c \gamma} r\right) \exp \left(\frac{i \omega}{\beta c} z\right) \\
& \widetilde{B}_{\phi}(r, z, \omega)=\frac{q \omega}{(2 \pi)^{3 / 2} \varepsilon_{0} \beta c^{3} \gamma} K_{1}\left(\frac{\omega}{\beta c \gamma} r\right) \exp \left(\frac{i \omega}{\beta c} z\right)
\end{aligned}
$$

Where $\beta$ and $\gamma$ are the relativistic parameters of the electron, $q$ is its charge, and $r$ and $z$ are the usual cylindrical coordinates as measured from the electron in the laboratory frame.

Using the approach above and formulae (2) - (4) as our source field at a given frequency, images of transition radiation (or coherent transition radiation if several fields from a charge distribution are used as the initial field) are now in the preliminary stages of analysis. An image of a $\gamma=500$ single electron impinging on a $50.8 \mathrm{~mm}$ diameter metallic foil is shown in Figure 4 at a wavelength of $0.5 \mathrm{~mm}$ for qualitative demonstration and proof of principle only. A vertical cut $19 \mathrm{~mm}$ from impact center shows ringing of intensity distribution due to finite foil and the skewed bilateral symmetry due to 45-degree foil inclination, in agreement with [2]. A more detailed analysis of results and direct comparison to analytical solutions are to be presented in the final thesis report.

\section{METHOD EVALUATION AND PLANS}

The strengths found in using this code include solving for arbitrary geometries, solving in near of far field regions, simple constraint to finite surfaces in any region of interest, and that it is seen to be energy conserving, allowing propagation between surfaces without loss of relative field strengths. However, it is computationally expensive, the foil material properties are neglected in the current model, a true realization of the code requires computation of many second order effects, and processing at wavelengths less than $0.3 \mathrm{~mm}$ is seen to require more data points being calculated to reach convergence.

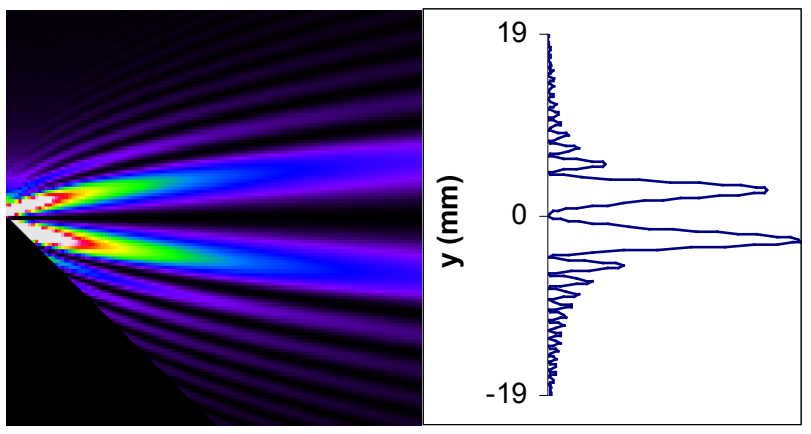

Figure 4: Single electron transition radiation intensity in transverse plane (arb. units), at $\lambda=0.5 \mathrm{~mm}$ for $\gamma=500$, in range extending to $38 \mathrm{~mm}$. Plotted only to $10 \%$ of $\max$ intensity for contrast (left). Vertical cut through center of image at $\mathrm{D}=19 \mathrm{~mm}$ (right).

With further developments of the method, full simulations are to be carried out mimicking experiments at the Fermilab/NICADD photoinjector and compared to experiment, assuming the problem remains approachable. Preliminary estimates at lowered data resolution and not including many of the optical elements show good agreement with other methods. However, basic benchmarking tests such as those presented here suggest that slight energy losses due to trimming of interference patterns just beyond the primary maximum may generate a frequency dependent response at the detector aperture of the interferometer. It is assumed for computational simplicity that radiation not in the acceptance region of the optical elements is scattered or absorbed and doesn't interfere in any regular way at the detecting surface. It is also worth noting that these effects have been seen to be strongest at longer wavelengths $(>0.7 \mathrm{~mm})$ where diffraction effects are more dominant. These longer wavelengths constitute the fringe of interest in typical CTR phenomena.

\section{REFERENCES}

[1] L. D. Landau and E. M. Lifschitz, Electrodynamics of Continuous Media, 2nd Edition, Pergaman Press, Oxford (1984).

[2] S. Casalbuoni, B. Schmidt and P. Schmüser, "FarInfrared Transition and Diffraction Radiation", TESLA Report 2005-15.

[3] C. A. Brau, Modern Problems in Classical Electrodynamics, Oxford University Press, Oxford (2004).

[4] A. S. Marathat and J. F. McCalmont, "Vector diffraction theory for electromagnetic waves", J. Opt. Soc. Am. A. 18, 2585-2593 (2001).

[5] T. J. Maxwell, Masters Thesis, Northern Illinois University, (In Preparation). 